\title{
ON SOME DIOPHANTINE RESULTS RELATED TO EULER POLYNOMIALS
}

\author{
CSABA RAKACZKI
}

\begin{abstract}
In this paper we prove that there are at most one complex number $b$ for which the shifted Euler polynomial $E_{n}(x)+b$ has at most two zeros of odd multiplicities.
\end{abstract}

\section{IntroduCtion}

Euler polynomials $E_{n}(x)$ are defined by the power series

$$
\frac{2 e^{x t}}{e^{t}+1}=\sum_{n=0}^{\infty} E_{n}(x) \frac{t^{n}}{n !} .
$$

The first fifteen Euler polynomials are listed for example in [16] on page 477. Euler polynomials appear in many classical results and play an important role in various approximation and expansion formulas which are applied both in numerical analysis and in analytic number theory (see [1], [12]). Numerous interesting and useful identities and properties involving Euler polynomials can be found in several publications (see for example [5], [8], [9], [14], [10], [17], [18]). Several authors investigated the values of Euler polynomials $E_{n}(x)$, where $x$ is a rational number. It can be shown that the Euler polynomials at rational arguments can be expressed in terms of a finite combination of trigonometric functions and the Hurwitz zeta function (see $[6]$ ).

Concerning the roots of Euler polynomials, Brillhart [3] proved that all Euler polynomials, except $E_{5}(x)$, have only simple roots. Further, the Euler polynomials have no rational root other than 0,1 , and 1/2. Later Delange [7], generalizing a result of Howard [11], gave an upper and a lower bound for the greatest real root of $E_{n}(x)$.

In the present paper we investigate the multiplicities of the zeros of shifted Euler polynomials $E_{n}(x)+b$, where $b$ is a complex number. We prove among other things that for given $n \geq 5$, there are at most one $b$ for which $E_{n}(x)+b$ has at most two zeros of odd multiplicities. Our result is an analogue of our earlier theorem [15] related to shifted Bernoulli polynomials. As an application we will give an effective finiteness theorem related to the hyperelliptic diophantine equation

$$
F\left(E_{n}(x)\right)=y^{2},
$$

where $F(x)$ is a polynomial with algebraic integer coefficients.

A polynomial $F(x)$ with complex coefficients will be called non-degenerate if it has at least three zeros of odd multiplicities and degenerate otherwise.

\section{Results}

For $n>1, L_{n}$ denotes the cardinality of the set of nonzero complex numbers $b$ for which $E_{n}(x)+b$ is degenerate, where $E_{n}(x)$ is the $n$th Euler polynomial.

2000 Mathematics Subject Classification. 11D41, $11 \mathrm{~B} 68$.

Key words and phrases. Euler polynomials, Higher degree equations.

This author was supported, in part, by Grants T48791 and F68872 form HNFSR, and by Hungarian Academy of Sciences. 
Theorem 1. We have $L_{3}=L_{4}=2$. Further, if $n \geq 5$ is an odd positive integer then $L_{n}=0$ while in case when $n \geq 6$ is even then $L_{n} \leq 1$.

The next result applies to hyperelliptic equations with algebraic integer coefficients. This is a simple consequence of Theorem 1 and Lemma 4 of Brindza [4].

Theorem 2. Let $\mathbb{K}$ be an algebraic number field with ring of integers $O_{\mathbf{K}}$, and let $F(x) \in$ $O_{\mathbf{K}}[x]$ be a non-square polynomial. Then the equation

$$
F\left(E_{n}(x)\right)=y^{2}
$$

has only finitely many solutions $x, y \in O_{\mathbf{K}}$ which can be effectively determined, provided that $n \geq 5$ and $\operatorname{deg} F(x)$ is even or $\operatorname{deg} F(x)$ and $n \geq 5$ are odd positive integers.

\section{Auxiliary Results}

The following are well-known identities of Euler polynomials:

Lemma 1. For a positive integer $n, E_{n}(x)$ denotes the $n$th Euler polynomial. Then we have

(i) $E_{n}^{\prime}(x)=n E_{n-1}(x)$.

(ii) $E_{n}(x)=(-1)^{n} E_{n}(1-x)$.

(iii) $E_{2 n}(0)=E_{2 n}(1)=0$.

(iv) The only rational root of $E_{2 n-1}(x)$ is $\frac{1}{2}$.

(v) $E_{5}(x)$ is the only Euler polynomial with a multiple root.

Proof. See [3].

Lemma 2. If $n$ is a rational integer with $n \geq 5$ and $a, b$ are complex numbers with $b \neq 0$ then the polynomial $\left(E_{n}(x)+a\right)^{2}+b$ is non-degenerate.

Proof. On supposing the contrary we have

$$
\left(E_{n}(x)+a\right)^{2}+b=f^{2}(x)
$$

or

$$
\left(E_{n}(x)+a\right)^{2}+b=g(x) f^{2}(x),
$$

for some $f(x), g(x) \in \mathbb{C}[x]$, where $g(x)$ is a quadratic polynomial with nonzero discriminant. From (3) we obtain that

$$
2\left(E_{n}(x)+a\right) E_{n}^{\prime}(x)=2 f(x) f^{\prime}(x) .
$$

However, since $\left(f(x), E_{n}(x)+a\right)=1$ we obtain that $f(x) \mid E_{n}^{\prime}(x)$, but then $n=\operatorname{deg} f(x) \leq$ $\operatorname{deg} E_{n}^{\prime}(x)=n-1$ which is impossible.

If we differentiate (4), we have

$$
2\left(E_{n}(x)+a\right) E_{n}^{\prime}(x)=2 g(x) f(x) f^{\prime}(x)+g^{\prime}(x) f^{2}(x)=f(x)\left(2 g(x) f^{\prime}(x)+g^{\prime}(x) f(x)\right) .
$$

Since $b \neq 0$, we obtain from (4) that $\left(f(x) g(x), E_{n}(x)+a\right)=1$, so

$$
f(x) \mid E_{n}^{\prime}(x) \text { and } E_{n}(x)+a \mid 2 g(x) f^{\prime}(x)+g^{\prime}(x) f(x) .
$$

It is easy to see that $\operatorname{deg} f(x)=n-1$, therefore $E_{n}^{\prime}(x)=c_{1} f(x)$ and $g^{\prime}(x) f(x)+2 g(x) f^{\prime}(x)=$ $c_{2}\left(E_{n}(x)+a\right)$, where $c_{1}, c_{2}$ are nonzero complex numbers. Substituting the first relation into the second one, we get

$$
\frac{1}{c_{1}} g^{\prime}(x) E_{n}^{\prime}(x)+\frac{2}{c_{1}} g(x) E_{n}^{\prime \prime}(x)=c_{2}\left(E_{n}(x)+a\right)
$$

and thus

$$
\frac{1}{c_{1}} g^{\prime \prime}(x) E_{n}^{\prime}(x)+\frac{3}{c_{1}} g^{\prime}(x) E_{n}^{\prime \prime}(x)+\frac{2}{c_{1}} g(x) E_{n}^{\prime \prime \prime}(x)=c_{2} E_{n}^{\prime}(x) \text {. }
$$


Applying (i) from Lemma 1, we infer that

(6) $\left(\frac{1}{c_{1}} g^{\prime \prime}(x)-c_{2}\right) E_{n-1}(x)+\frac{3(n-1)}{c_{1}} g^{\prime}(x) E_{n-2}(x)+\frac{2(n-1)(n-2)}{c_{1}} g(x) E_{n-3}(x)=0$.

For odd values of $n$ we substitute $x=0,1$ into (6) and we obtain, by (iii) of Lemma 1 , that

$$
g^{\prime}(0) E_{n-2}(0)=g^{\prime}(1) E_{n-2}(1)=0 .
$$

Since $g^{\prime}(x)$ is a linear polynomial, thus $E_{n-2}(0) E_{n-2}(1)=0$. But this contradicts (iv) from Lemma 1.

In case when $n$ is even, differentiating (6) and using again (i) from Lemma 1, we have

(7) $\left(\frac{4}{c_{1}} g^{\prime \prime}(x)-c_{2}\right) E_{n-2}(x)+\frac{5(n-2)}{c_{1}} g^{\prime}(x) E_{n-3}(x)+\frac{2(n-2)(n-3)}{c_{1}} g(x) E_{n-4}(x)=0$,

and the same argument as in the odd case gives a contradiction again.

Lemma 3. Let $f(x)$ be a polynomial with complex coefficients. If $\operatorname{deg} f(x) \geq 5$ then there are at most two complex numbers $b$ for which the polynomial $f(x)+b$ is degenerate.

Proof. On supposing the contrary we have

$$
f(x)+b_{i}=g_{i}(x)\left(h_{i}(x)\right)^{2} \quad i=1,2,3,
$$

where the polynomials $g_{i}(x) \in \mathbb{C}[x] i=1,2,3$ are of degree at most 2 with nonzero discriminant, $h_{i}(x) \in \mathbb{C}[x] i=1,2,3$. One can see from (8) that

$$
\left(h_{1}(x), h_{2}(x)\right)=\left(h_{1}(x), h_{3}(x)\right)=\left(h_{2}(x), h_{3}(x)\right)=1 .
$$

From (8) we obtain that $\operatorname{deg} h_{i}(x) \geq(\operatorname{deg} f(x)-2) / 2, i=1,2,3$. Now, on differentiating equation (8) we deduce that

$$
f^{\prime}(x)=g_{i}^{\prime}(x)\left(h_{i}(x)\right)^{2}+2 g_{i}(x) h_{i}(x) h_{i}^{\prime}(x) \quad i=1,2,3 .
$$

It follows from this that $h_{i}(x) \mid f^{\prime}(x) i=1,2,3$. Using (9) one can observe that

$$
h_{1}(x) h_{2}(x) h_{3}(x) \mid f^{\prime}(x)
$$

and so $\operatorname{deg} h_{1}(x)+\operatorname{deg} h_{2}(x)+\operatorname{deg} h_{3}(x) \leq \operatorname{deg} f^{\prime}(x)$. Since $\operatorname{deg} h_{i}(x) \geq(\operatorname{deg} f(x)-2) / 2$ $i=1,2,3$ we have that

$$
3 \frac{\operatorname{deg} f(x)-2}{2} \leq \operatorname{deg} f(x)-1
$$

that is $\operatorname{deg} f(x) \leq 4$ which is a contradiction.

Several authors investigated the superelliptic equation $f(x)=y^{m}$, where $f(x)$ is a polynomial of degree $\geq 3$ with integer or algebraic integer coefficients and $m \in \mathbb{N}$. LeVeque [13] gave a criterion for superelliptic equations to have only finitely many integer solutions. LeVeque's theorem is ineffective. Baker [2] was the first to prove a general effective result for the solutions of the equation $f(x)=y^{m}$. Since then a lot of generalizations and extensions of Baker's result have been established. The following result is an effective version of LeVeque's theorem.

Let $\mathbb{K}$ be an algebraic number field with ring of integers $O_{\mathbf{K}}$.

Lemma 4. (Brindza, [4]) Let

$$
f(x)=a_{0} x^{N}+\cdots+a_{N}=a_{0} \prod_{i=1}^{n}\left(x-\alpha_{i}\right)^{r_{i}}
$$

be a polynomial in $O_{\mathbf{K}}[x]$ with $a_{0} \neq 0$ and $\alpha_{i} \neq \alpha_{j}$ for $i \neq j$. Further, let $b \in O_{\mathbf{K}}, m>1$ and $q_{i}=m /\left(m, r_{i}\right), i=1,2, \cdots, n$. Suppose that $\left(q_{1}, q_{2}, \cdots, q_{n}\right)$ is not a permutation of $(q, 1, \cdots, 1)$ or $(2,2,1, \cdots, 1)$, where $q \geq 1$. Then the equation

$$
f(x)=b y^{m} \quad \text { in } \quad x, y \in O_{\mathbf{K}}
$$


has only finitely many solutions and all these can be effectively determined.

An easy consequence of this result is that the hyperelliptic equation

$$
f(x)=y^{2} \text { in } x, y \in O_{\mathbf{K}}
$$

has only finitely many solutions $x, y$ provided that the polynomial $f(x)$ is non-degenerated.

\section{Proofs}

Proof of Theorem 1. For small values of $n$ we can use the following observation: the discriminant of $E_{n}(x)+b$ is a polynomial in $b$ of degree $n-1$. Hence apart from at most $n-1$ distinct values of $b$ the polynomial $E_{n}(x)+b$ has only simple zeros. From Lemma 3 we know that there are at most two complex numbers for which the shifted Euler polynomials are degenerate. Suppose that $b_{1}$ and $b_{2}$ are two distinct complex numbers for which $E_{n}(x)+b_{i}$ are degenerate $i=1,2$. If $n \geq 5$ is an odd integer then we have

$$
E_{n}(x)+b_{i}=g_{i}(x) f_{i}^{2}(x)
$$

where $f_{i}(x), g_{i}(x) \in \mathbb{C}[x]$ and $\operatorname{deg} g_{i}(x)=1$ for $i=1,2$. We know that $b_{1} \neq b_{2}$, hence $\left(g_{2}(x), f_{1}(x)\right)=\left(g_{1}(x), f_{2}(x)\right)=\left(g_{1}(x), g_{2}(x)\right)=1$. It is clear that the polynomial

$$
\begin{aligned}
\left(E_{n}(x)+b_{1}\right)\left(E_{n}(x)+b_{2}\right)=\left(E_{n}(x)+\frac{b_{1}+b_{2}}{2}\right)^{2}-\left(\frac{b_{1}+b_{2}}{2}\right)^{2}+b_{1} b_{2}= \\
=g_{1}(x) g_{2}(x)\left(f_{1}(x) f_{2}(x)\right)^{2}
\end{aligned}
$$

is degenerate. However, it follows from Lemma 2 that $b_{1} b_{2}-\left(\frac{b_{1}+b_{2}}{2}\right)^{2}=0$. But then $b_{1}=b_{2}=0$, which is a contradiction.

Now suppose that there is a nonzero value $b$ such that the polynomial $E_{n}(x)+b$ is degenerate. Then it is easy to see that the polynomial $E_{n}(1-x)+b$ is also degenerate. It follows from (ii) of Lemma 1 that $-E_{n}(x)+b$ and so $E_{n}(x)-b$ are also degenerate. Since $b$ is nonzero, we obtain two distinct values, $b$ and $-b$, for which the polynomials $E_{n}(x) \pm b$ are degenerate, which is impossible.

Assume now that $n$ is even. In this case there are the following four possibilities:

(a) $E_{n}(x)+b_{1}=f_{1}(x)^{2}$ and $E_{n}(x)+b_{2}=f_{2}(x)^{2}$,

(b) $E_{n}(x)+b_{1}=f_{1}(x)^{2}$ and $E_{n}(x)+b_{2}=g_{2}(x) f_{2}(x)^{2}$,

(c) $E_{n}(x)+b_{1}=g_{1}(x) f_{1}(x)^{2}$ and $E_{n}(x)+b_{2}=f_{2}(x)^{2}$,

(d) $E_{n}(x)+b_{1}=g_{1}(x) f_{1}(x)^{2}$ and $E_{n}(x)+b_{2}=g_{2}(x) f_{2}(x)^{2}$,

where the polynomials $g_{1}(x), g_{2}(x) \in \mathbb{C}$ are quadratic polynomials with nonzero discriminant, $f_{1}(x), f_{2}(x) \in \mathbb{C}[x]$. Moreover, $g_{1}(x), g_{2}(x), f_{1}(x), f_{2}(x)$ are pairwise coprime polynomials in $\mathbb{C}[x]$. In cases $(a),(b)$ and $(c)$ we can deduce again that the polynomial

$$
\left(E_{n}(x)+b_{1}\right)\left(E_{n}(x)+b_{2}\right)=\left(E_{n}(x)+\frac{b_{1}+b_{2}}{2}\right)^{2}-\left(\frac{b_{1}+b_{2}}{2}\right)^{2}+b_{1} b_{2}
$$

is degenerate. However, from Lemma 2 we obtain that $\left(\left(b_{1}+b_{2}\right) / 2\right)^{2}-b_{1} b_{2}=0$ and so $b_{1}=b_{2}$. Consider now the last case $(d)$. We can suppose that

$$
g_{1}(x)=\left(x-\alpha_{1}\right)\left(x-\alpha_{2}\right) \text { and } f_{1}(x)=\prod_{i=1}^{n / 2-1}\left(x-\beta_{i}\right)
$$

and

$$
g_{2}(x)=\left(x-\gamma_{1}\right)\left(x-\gamma_{2}\right) \text { and } f_{2}(x)=\prod_{i=1}^{n / 2-1}\left(x-\delta_{i}\right),
$$

where $\alpha_{j}, \gamma_{j}, \beta_{i}, \delta_{i} \in \mathbb{C}, j=1,2, i=1, \ldots, n / 2-1$ and $\alpha_{1} \neq \alpha_{2}, \gamma_{1} \neq \gamma_{2}$. 
Using (ii) from Lemma 1 we infer that

$$
\left(x-\alpha_{1}\right)\left(x-\alpha_{2}\right) \prod_{i=1}^{n / 2-1}\left(x-\beta_{i}\right)^{2}=\left(1-x-\alpha_{1}\right)\left(1-x-\alpha_{2}\right) \prod_{i=1}^{n / 2-1}\left(1-x-\beta_{i}\right)^{2}
$$

that is

$$
\left(x-\alpha_{1}\right)\left(x-\alpha_{2}\right) \prod_{i=1}^{n / 2-1}\left(x-\beta_{i}\right)^{2}=\left(x-\left(1-\alpha_{1}\right)\right)\left(x-\left(1-\alpha_{2}\right)\right) \prod_{i=1}^{n / 2-1}\left(x-\left(1-\beta_{i}\right)\right)^{2} .
$$

One can see from (15) that

$$
\alpha_{1}, \alpha_{2} \in\left\{1-\alpha_{1}, 1-\alpha_{2}, 1-\beta_{1}, \ldots, 1-\beta_{n / 2-1}\right\} .
$$

Suppose that $\alpha_{1}=1-\beta_{j}$ for some $j \in\{1,2, \ldots, n / 2-1\}$. Then $x-\alpha_{1}=x-\left(1-\beta_{j}\right)$ and

(16) $\left(x-\alpha_{2}\right) \prod_{i=1}^{n / 2-1}\left(x-\beta_{i}\right)^{2}=\left(x-\left(1-\alpha_{1}\right)\right)\left(x-\left(1-\alpha_{2}\right)\right)\left(x-\left(1-\beta_{j}\right)\right) \prod_{i=1, i \neq j}^{n / 2-1}\left(x-\left(1-\beta_{i}\right)\right)^{2}$.

Hence $1-\beta_{j} \in\left\{\alpha_{2}, \beta_{1}, \ldots, \beta_{n / 2-1}\right\}$. If $1-\beta_{j}=\alpha_{2}$ then $\alpha_{1}=\alpha_{2}$ which contradicts our assumption that $g_{1}(x)$ has non-zero discriminant. Thus $\alpha_{1}=1-\beta_{j}=\beta_{k}$ for some $k \in\{1, \ldots, n / 2-1\}$. However, in this case $\left(g_{1}(x), f_{1}(x)\right) \neq 1$ and so $E_{n}^{\prime}(x)=n E_{n-1}(x)$ has a multiple root, but it is possible only in case when $n=6$ by (v) from Lemma 1 . It follows from the above that

$$
\alpha_{1}, \alpha_{2} \in\left\{1-\alpha_{1}, 1-\alpha_{2}\right\} .
$$

If $\alpha_{1}=1-\alpha_{1}$ then $\alpha_{2}=1-\alpha_{2}$ and $\alpha_{1}=\alpha_{2}=1 / 2$ which is impossible. So we have

$$
\alpha_{1}+\alpha_{2}=1 \text { and } \beta_{j} \in\left\{1-\beta_{1}, \ldots, 1-\beta_{n / 2-1}\right\} j=1, \ldots, n / 2-1 \text {. }
$$

Using the same argument as above we can infer that

$$
\gamma_{1}+\gamma_{2}=1 \text { and } \delta_{j} \in\left\{1-\delta_{1}, \ldots, 1-\delta_{n / 2-1}\right\} j=1, \ldots, n / 2-1 .
$$

If $n / 2-1$ is odd then this implies that $\frac{1}{2} \in\left\{\beta_{1}, \beta_{2}, \ldots \beta_{n / 2-1}\right\} \bigcap\left\{\delta_{1}, \ldots, \delta_{n / 2-1}\right\}$. But this gives a contradiction because then $f_{1}(1 / 2)=f_{2}(1 / 2)=0$ and so $b_{1}=b_{2}$.

Suppose that $n / 2-1$ is even. Then $f_{1}(1 / 2) f_{2}(1 / 2) \neq 0$. Otherwise, we can see from (17) or $(18)$ that $1 / 2$ is a multiple root of the polynomial $f_{1}(x)$ or $f_{2}(x)$. Then the polynomial $E_{n}^{\prime}(x)=n E_{n-1}(x)$ has a multiple root by $(d)$. But, by Lemma 1 , this is possible only if $n=6$. From $(d)$ we obtain that

$$
\left(E_{n}(x)+a\right)^{2}+b=g(x) f(x)^{2},
$$

where

$$
a=\frac{b_{1}+b_{2}}{2}, b=b_{1} b_{2}-\left(\frac{b_{1}+b_{2}}{2}\right)^{2}, f(x)=f_{1}(x) f_{2}(x)
$$

and

$$
g(x)=\left(x^{2}-x+c_{1}\right)\left(x^{2}-x+c_{2}\right)
$$

where $c_{1}=\alpha_{1} \alpha_{2}, c_{2}=\gamma_{1} \gamma_{2}$. One can observe from (19) that

$$
2\left(E_{n}(x)+a\right) E_{n}^{\prime}(x)=f(x)\left(g^{\prime}(x) f(x)+2 g(x) f^{\prime}(x)\right) .
$$

Since $\left(E_{n}(x), f(x)\right)=1$, therefore $f(x) \mid E_{n-1}(x)$. This fact and property (iv) of Lemma 1 yield that

$$
E_{n-1}(x)=\left(x-\frac{1}{2}\right) f(x)
$$

further,

$$
2 n\left(E_{n}(x)+a\right)\left(x-\frac{1}{2}\right)=g^{\prime}(x) f(x)+2 g(x) f^{\prime}(x) .
$$

One can observe from Lemma 1 and (22) that 
(24) $(n-1)(n-2) \cdots(n-(i+1)) E_{n-(i+2)}(x)=$

$$
=f^{(i+1)}(x)\left(x-\frac{1}{2}\right)+(i+1) f^{(i)}(x), i=0,1, \ldots, n-2 .
$$

If we substitute $x=1 / 2$ into (24) we obtain that

$$
f^{(i)}\left(\frac{1}{2}\right)= \begin{cases}0, & \text { if } i \text { is odd } \\ \frac{1}{i+1}(n-1)(n-2) \cdots(n-(i+1)) E_{n-(i+2)}\left(\frac{1}{2}\right), & \text { if } i \text { is even. }\end{cases}
$$

From relations (20) and (21) we deduce the following:

(26) $g\left(\frac{1}{2}\right)=\frac{1}{16}-\frac{1}{4}\left(c_{1}+c_{2}\right)+c_{1} c_{2}, g^{\prime}\left(\frac{1}{2}\right)=g^{\prime \prime \prime}\left(\frac{1}{2}\right)=0, g^{\prime \prime}\left(\frac{1}{2}\right)=-1+2\left(c_{1}+c_{2}\right)$.

After the $i-1 \geq 3$-th derivation from (23) we obtain that

$$
\begin{aligned}
2 n^{2}(n-1) \cdots(n-(i-3))\left[(n-(i-2)) E_{n-(i-1)}(x)\left(x-\frac{1}{2}\right)\right. & \left.+(i-1) E_{n-(i-2)}(x)\right]= \\
& =\sum_{j=0}^{4} a_{i j} g^{(j)}(x) f^{(i-j)}(x),
\end{aligned}
$$

where

$$
a_{i 0}=2, a_{i 1}=2 i-1, a_{i 2}=(i-1)^{2}, a_{i 3}=\frac{(i-1)(i-2)(2 i-3)}{6}
$$

and

$$
a_{i 4}=\frac{(i-1)(i-2)^{2}(i-3)}{12} .
$$

Substitute $i=n, n-2, n-4$ and $x=1 / 2$ into (27). Using (25), (26) and $\operatorname{deg} f(x)=n-2$ we obtain that

(28) $2 n^{2}(n-1)(n-2) \cdots 3(n-1) E_{2}\left(\frac{1}{2}\right)=$

$$
\begin{aligned}
=(n-1)^{2}( & \left.-1+2\left(c_{1}+c_{2}\right)\right) \frac{1}{n-1}(n-1)(n-2) \cdots 2+ \\
& +2(n-1)(n-2)^{2}(n-3) \frac{1}{n-3}(n-1)(n-2) \cdots 3 E_{2}\left(\frac{1}{2}\right),
\end{aligned}
$$

(29) $2 n^{2}(n-1)(n-2) \cdots 5(n-3) E_{4}\left(\frac{1}{2}\right)=$

$$
\begin{aligned}
=2\left(\frac{1}{16}-\right. & \left.\frac{1}{4}\left(c_{1}+c_{2}\right)+c_{1} c_{2}\right) \frac{1}{n-1}(n-1)(n-2) \cdots 2+ \\
+(n-3)^{2}(-1 & \left.+2\left(c_{1}+c_{2}\right)\right) \frac{1}{n-3}(n-1)(n-2) \cdots 3 E_{2}\left(\frac{1}{2}\right)+ \\
& +2(n-3)(n-4)^{2}(n-5) \frac{1}{n-5}(n-1)(n-2) \cdots 5 E_{4}\left(\frac{1}{2}\right)
\end{aligned}
$$


(30)

$$
\begin{aligned}
& 2 n^{2}(n-1)(n-2) \cdots 7(n-5) E_{6}\left(\frac{1}{2}\right)= \\
& =2\left(\frac{1}{16}-\frac{1}{4}\left(c_{1}+c_{2}\right)+c_{1} c_{2}\right) \frac{1}{n-3}(n-1)(n-2) \cdots 3 E_{2}\left(\frac{1}{2}\right)+ \\
& \quad+(n-5)^{2}\left(-1+2\left(c_{1}+c_{2}\right)\right) \frac{1}{n-5}(n-1)(n-2) \cdots 5 E_{4}\left(\frac{1}{2}\right)+ \\
& \quad+2(n-5)(n-6)^{2}(n-7) \frac{1}{n-7}(n-1)(n-2) \cdots 7 E_{6}\left(\frac{1}{2}\right),
\end{aligned}
$$

We know that

$$
E_{2}\left(\frac{1}{2}\right)=-\frac{1}{4}, E_{4}\left(\frac{1}{2}\right)=\frac{5}{16}, E_{6}\left(\frac{1}{2}\right)=-\frac{61}{64} .
$$

We can deduce from (28) and (29) that

$$
\begin{gathered}
c_{1}+c_{2}=\frac{-n+2}{2} \\
c_{1} c_{2}=\frac{(n-2)\left(2 n^{2}-11 n+6\right)}{48} .
\end{gathered}
$$

Using these relations from (30) we get the following equation:

$$
-6 n^{3}+111 n^{2}-549 n+810=0 .
$$

It is easy to see that this equation has only one integer solution $n=3$.

Proof of Theorem 2. Let $\mathbb{K}$ be an algebraic number field with ring of integers $O_{\mathbf{K}}$ and let

$$
F(x)=\prod_{i=1}^{t}\left(x-\alpha_{i}\right)^{k_{i}} \in O_{\mathbf{K}}[x]
$$

be a non-square polynomial. If $\operatorname{deg} F(x)$ is odd then we can suppose that $k_{1}$ is also odd. Since $E_{n}(x)$ is non-degenerate, providing that $n \geq 5$ is odd, therefore the polynomial

$$
F\left(E_{n}(x)\right)=\prod_{i=1}^{t}\left(E_{n}(x)-\alpha_{i}\right)^{k_{i}}, \quad n \geq 5 \text { odd },
$$

is also non-degenerate.

In case when $\operatorname{deg} F(x) \geq 6$ is even we can assume that $k_{1}$ and $k_{2}$ are odd.

We know from Theorem 1 that there is at most one complex number $b$ for which the shifted Euler polynomial $E_{n}(x)+b$ is degenerate. It follows from this and

$$
F\left(E_{n}(x)\right)=\prod_{i=1}^{t}\left(E_{n}(x)-\alpha_{i}\right)^{k_{i}}
$$

that the polynomial $F\left(E_{n}(x)\right)$ is non-degenerate. Hence our statement is true by Lemma 4 .

\section{REFERENCES}

[1] M. Abramowitz and I. Stegun (eds.), Handbook of mathematical functions with formulas, graphs and mathematical tables, Dover, New York, 1972.

[2] A. Baker, Bounds for the solutions of the hyperelliptic equation, Proc. Camb. Phil. Soc. 65 (1969), 439-444.

[3] J. Brillhart, On the Euler and Bernoulli polynomials, J. Reine Angew. Math. 234 (1969), 45-64.

[4] B. Brindza, On S-integral solutions of the equation $y^{m}=f(x)$, Acta. Math. Hung. 44 (1984), 133-139.

[5] G.-S. Cheon, A note on the Bernoulli and Euler polynomials, Appl. Math. Lett. 16 (2003), 365-368.

[6] D. Cvijović and J. Klinowski, New formulae for the Bernoulli and Euler polynomials at rational arguments, Proc. Amer. MAth. Soc. 123 (1995), 1527-1535. 
[7] H. Delange, On the real roots of Euler polynomials, Monatsh.Math. 106 (1988), 115-138.

[8] A. Erdélyi, W. Magnus, F. Oberhettinger and F. G. Tricomi, Higher Transcendental Functions, Volume I, McGraw-Hill, New York, (1953).

[9] E. R. Hansen, A Table of Series and Products, Prentice Hall, Englewood Cliffs, NJ, (1975).

[10] Hao Pan and Zhi-Wei Sun, New identities involving Bernoulli and Euler polynomials, J. Combin. Theory Ser. A 113 (2006), 156-175.

[11] F. T. Howard, Roots of The Euler polynomials, Pacific J. Math. 64 (1976), 181-191.

[12] C. Jordan, Calculus of finite differences, Chelsea, New York, 1947.

[13] W. J. LeVeque, On the equation $y^{m}=f(x)$, Acta Arith. 9 (1964), 209-219.

[14] W. Magnus, F. Oberhettinger and R. P. Soni, Formulas and Theorems for the Special Functions of Mathematical Physics, Third Enlarged Edition, Springer-Verlag, New York, (1966).

[15] Á. Pintér and Cs. Rakaczki. On the zeros of shifted Bernoulli polynomials, Appl. Math. Comput. 187 (2007), 379-393.

[16] S. Selby, editor in chief, CRC Standard Mathematical Tables, 17th Edition, The Chemical Rubber Co., Cleveland, 1969.

[17] H. M. Srivastava and J. Choi, Series Associated with the Zeta and Related Functions, Kluwer Academic, Dordrecht, (2001).

[18] H. M. Srivastava and Á. Pintér, Remarks on some relationships between the Bernoulli and Euler Polynomials, Appl. Math. Lett. 17 (2004), 375-380.

Number Theory Research Group of the Hungarian Academy of Sciences

Institute OF MATHEMATICS,

UNIVERSITY OF DEBRECEN

H-4010 Debrecen, P.O.B. 12

Hungary

E-mail address: rcsaba@math.klte.hu 